\title{
Nanoscale
}

D) Check for updates

Cite this: Nanoscale, 2022, 14, 3062

\section{Widefield phototransient imaging for visualizing 3D motion of resonant particles in scattering environments $\uparrow$}

\author{
Matz Liebel, (D) *a Franco V. A. Camargo, (iD b Giulio Cerullo iD ${ }^{\text {b,c }}$ and \\ Niek F. van Hulst iD *a,d
}

\begin{abstract}
Identifying, visualising and ultimately tracking dynamically moving non-fluorescent nanoparticles in the presence of non-specific scattering is a long-standing challenge across the nano- and life-sciences. In this work we demonstrate that our recently developed ultrafast holographic transient (UHT) microscope is ideally suited for meeting this challenge. We show that UHT microscopy allows reliably distinguishing offresonant, dielectric, from resonant, metallic, nanoparticles, based on the phototransient signal: a prerequisite for single-particle tracking in scattering environments. We then demonstrate the capability of UHT microscopy to holographically localize in 3D single particles over large volumes of view. Ultimately, we combine the two concepts to simultaneously track several tens of freely diffusing gold nanoparticles, within a $110 \times 110 \times 110 \mu \mathrm{m}$ volume of view at an integration time of $10 \mathrm{~ms}$ per frame, while simultaneously recording their phototransient signals. The combined experimental concepts outlined and validated in this work lay the foundation for background-free 3D single-particle tracking applications or spectroscopy in scattering environments and are immediately applicable to systems as diverse as live cells and tissues or supported heterogeneous catalysts.
\end{abstract}

Received 15th October 2021 Accepted 16th November 2021 DOI: $10.1039 / \mathrm{d} 1 \mathrm{nr} 06837 \mathrm{~g}$ rsc.li/nanoscale
When combined with femtosecond pulses, this so-called ultrafast holographic transient (UHT) microscope allowed us to widefield-record pump-probe time-delay dependent transient dynamics of a variety of gold nanoparticles (NPs) with diameters ranging from 20 to $200 \mathrm{~nm}$. Further, the holographic aspect of our approach allowed computational image propagation and refocussing.

In this article we show that UHT microscopy is ideally suited for 3D single-particle tracking, even in its most challenging form: the simultaneous observation of many freely diffusing NPs in large 3D volumes of observation. ${ }^{11}$ We first demonstrate that it is possible to discriminate resonant, metallic, from off-resonant, dielectric, NPs with comparable scattering signals, using 2D samples with immobilised particles. Next, we demonstrate that UHT signals are independent of the nominal sample-defocus by using static, pseudo-3D samples, in the form of 2D immobilised NPs that we place at precisely defined $z$-positions with respect to the image plane. Finally, we address dynamically moving systems by performing UHTbased tracking and time-dependent transient spectroscopy of freely diffusing Au NPs in large, $110 \times 110 \times 110 \mu \mathrm{m}$, volumes of observation.

Fig. 1 introduces the basic concept of UHT, which we only briefly describe here, referring the interested reader to our 


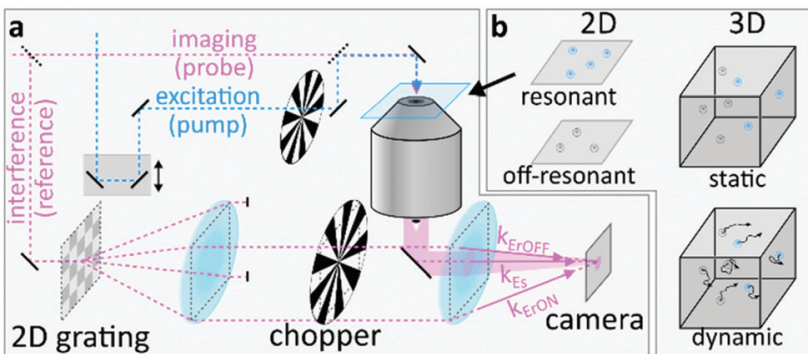

Fig. 1 UHT microscopy applied to a variety of samples. (a) Simplified schematic of an UHT microscope: a multiplexed off-axis holographic microscope is equipped with an additional ultrafast pump laser to selectively photoexcite the sample of interest. (b) Summary of typical samples discussed in this work.

proof-of-concept work ${ }^{10}$ for a detailed discussion of the more technical and spectroscopy-related aspects. Fig. 1a shows a schematic of an UHT microscope ${ }^{10}$ a multiplexed off-axis holographic imaging system ${ }^{12}$ which is modified to allow generating, demodulating and, ultimately, detecting photoinduced signals with ultrafast pulses (Methods). Briefly, a probe beam illuminates the sample which is imaged onto a conventional CMOS camera in either a dark-field or a bright-field configuration. A 2D $0-\pi$ phase grating, conjugate with the camera plane, generates two reference waves which interfere at different angles with the signal scattered/transmitted by the sample. The system outlined above is similar to a conventional multiplexed off-axis holographic microscope ${ }^{13}$ which allows using arbitrarily broadband pulses, as the grating ensures interference across the entire camera chip. ${ }^{12,14-17}$ To record photoinduced signals, an additional pump pulse illuminates the sample at a variable, but precisely controlled, pump-probe time-delay. Rather than recording separate sample images in the presence (pumpon) or absence (pumpofF) of the pump pulse, the UHT microscope simultaneously records the pump $_{\mathrm{ON}}$ and pump $\mathrm{PFF}_{\mathrm{O}}$ images in a single camera exposure via a multiplexed holographic demodulation scheme. ${ }^{10}$

The recorded image, $I_{\mathrm{UHT}}(x, y)$, can be described as:

$$
\begin{aligned}
I_{\mathrm{UHT}}(x, y)= & E_{\mathrm{s}}^{2}(x, y)+E_{\mathrm{rON}}^{2}(x, y) \\
& +E_{\mathrm{rOFF}}^{2}(x, y)+E_{s} E_{\mathrm{rON}}^{*}(x, y) e^{-i(\mathrm{a} x-\mathrm{b} y)} \\
& +E_{s} E_{\mathrm{rOFF}}^{*}(x, y) e^{-i(\mathrm{a} x+\mathrm{b} y)}+c c .,
\end{aligned}
$$

with $(x, y)$ denoting the camera pixels, $E_{\mathrm{s}}$ the signal field generated by propagating the probe-field through the sample and the imaging system, $E_{\mathrm{rOFF}}$ and $E_{\mathrm{rON}}$ the reference waves in the absence and presence of the pump pulse, $c c$. denotes the complex conjugate terms. The camera position dependent linear phase terms, $a x$ and by, are a direct result of the intentionally non-collinear wave-vectors of $E_{\mathrm{s}}, E_{\mathrm{rOFF}}$ and $E_{\mathrm{rON}}$ (Fig. 1a). Ultimately, these terms enable the well-established Fourier filtering-based approach to off-axis hologram processing. ${ }^{18,19}$ As illustrated in Fig. 1b, UHT microscopy is very flexible and can be applied to resonant and off-resonant samples, in 2D and 3D, in both static and dynamic configurations.

Fig. 2a shows a typical UHT image, acquired for $80 \mathrm{~nm} \mathrm{Au}$ NPs in dark-field configuration, alongside its Fourier transformation. Interference between the probe and the reference fields directly manifests itself as oscillatory modulation of the NPs' point-spread-functions (PSFs). As mentioned above, a spatial Fourier transformation allows selectively isolating the interference terms $E_{\mathrm{S}} E_{\mathrm{rON}}^{*}$ and $E_{\mathrm{S}} E_{\mathrm{rOFF}}^{*}$ in the Fourier plane followed by removal of the linear phase ramp and an inverse Fourier transformation..$^{10,18,19}$

To selectively encode the image-information recorded in the presence (absence) of the pump pulse into $E_{\mathrm{S}} E_{\mathrm{rON}}^{*}\left(E_{\mathrm{S}} E_{\mathrm{rOFF}}^{*}\right)$, we modulate both the pump beam and the reference waves with optical choppers (Fig. 2b). The camera detects multiple probe pulses, within a single exposure, which interrogate the sample both with and without pump excitation. The synchronised pump and reference modulation ensures that the $E_{\mathrm{rON}}$ $\left(E_{\mathrm{rOFF}}\right)$ reference wave only interferes with probe pulses that interrogate the sample in the presence (absence) of the pump pulse. This all-optical lock-in camera can, in principle, operate at arbitrarily fast demodulation frequencies which are independent of the CMOS camera frame rate, as the demodulation purely relies on computational hologram postprocessing and not on fast electronics. As such, the alloptical widefield lock-in modality of the UHT microscope conveniently eliminates the need for point scanning, which is necessary when operating with single-pixel-detector-based lock-in amplifiers, thus making UHT microscopy an ideal candidate for widefield observations of dynamic scenes.

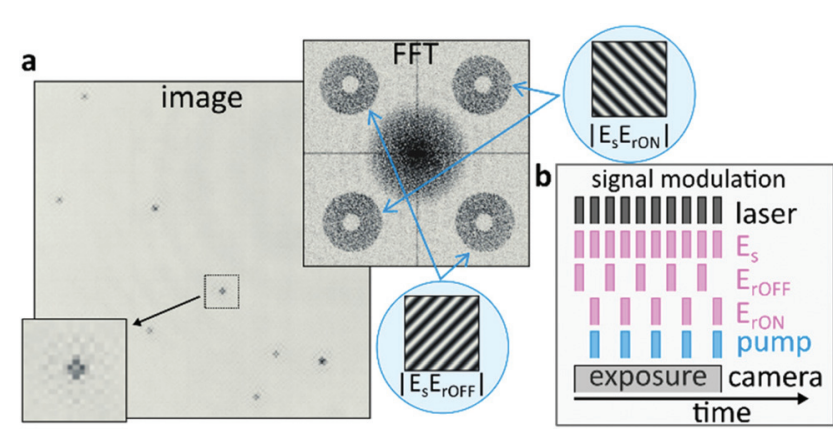

Fig. 2 Image processing and signal demodulation in UHT microscopy. (a) Typical UHT image of multiple $80 \mathrm{~nm}$ Au NPs alongside its Fourier transformation and a magnification of a point-spread-function to highlight the interference pattern. The central part of the Fourier transformation (DC) contains the amplitude square terms shown in eqn (1). The four terms along the diagonals are the respective interference terms which are displaced as a result of the linear phase terms. The cartoon insets visualise how linear phase terms would manifest themselves in the most intuitive case of interference between two plane waves. (b) UHT modulation scheme that allows encoding and retrieving the desired information from a single camera exposure. Each "bar" represents a laser pulse but the modulation scheme is equally applicable to CW-illumination. 
A key asset of UHT microscopy in the context of biological or biomedical imaging is its capability to discriminate resonant particles from the off-resonant scattering background which might arise from cells or tissue..$^{20}$ To explore the applicability of UHT microscopy to such scenarios, we compare UHT signals obtained for $60 \mathrm{~nm} \mathrm{Au}$ and $100 \mathrm{~nm}$ latex spheres that we deposit on bare coverglass (Fig. 3a). The holographically recorded scattering amplitude of both sets of particles is similar (Fig. 3b), thus making them essentially indistinguishable when measured in a linear colour-blind fashion. ${ }^{12}$ Their pump-probe time-delay dependent UHT response, however, differs considerably as can be seen by evaluating the scattering signal change computed as the difference between images acquired in the presence and absence of the pump pulse (Fig. 3c). The latex NPs show no transient signal, as expected for off-resonantly excited particles probed far off-resonance (Fig. 3d). Contrary, the Au NPs exhibit an instrument-response limited decrease in differential scattering signal, $\Delta S / S$, due to the generation of hot electrons ${ }^{21}$ followed by thermalisation on the picosecond time-scale. We note that the $\Delta S / S$ signaldifferences between individual NPs are due to the Gaussian spatial profile of the pump beam, which only weakly photoexcites NPs positioned at its wings.

Beyond being able to simultaneously observe, and identify, many individual nano-objects within a large observation plane, the holographic nature of our method furthermore allows computational image propagation, post-acquisition, to 3D-localise many individual particles from a single camera
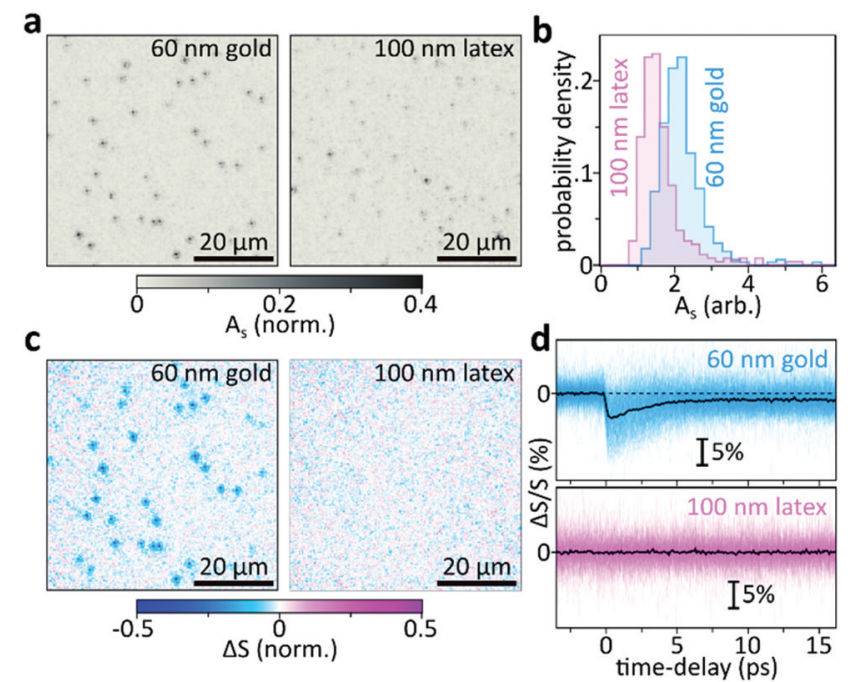

Fig. 3 Transient scattering differentiates resonant and off-resonant particles. (a) Representative holographic scattering images of $60 \mathrm{~nm} \mathrm{Au}$ and $100 \mathrm{~nm}$ latex beads. (b) Typical steady-state scattering amplitudes $\left(A_{s}\right)$ obtained by evaluating the individual scattering signals of 266 latex and 337 gold NPs. (c) Normalised scattering signal change for the particles shown in (a). The image is an average over the pump-probe delay window between 0-1.6 ps (15 images averaged). Both images are normalised to the same max/min amplitudes to allow directly comparing the signal magnitudes. (d) Pump-probe delay dependent transient scattering signals for all particles evaluated, 337 (gold) and 266 (latex) traces are shown. Pump: $400 \mathrm{~nm}$, probe: $550 \mathrm{~nm}$ wavelength.

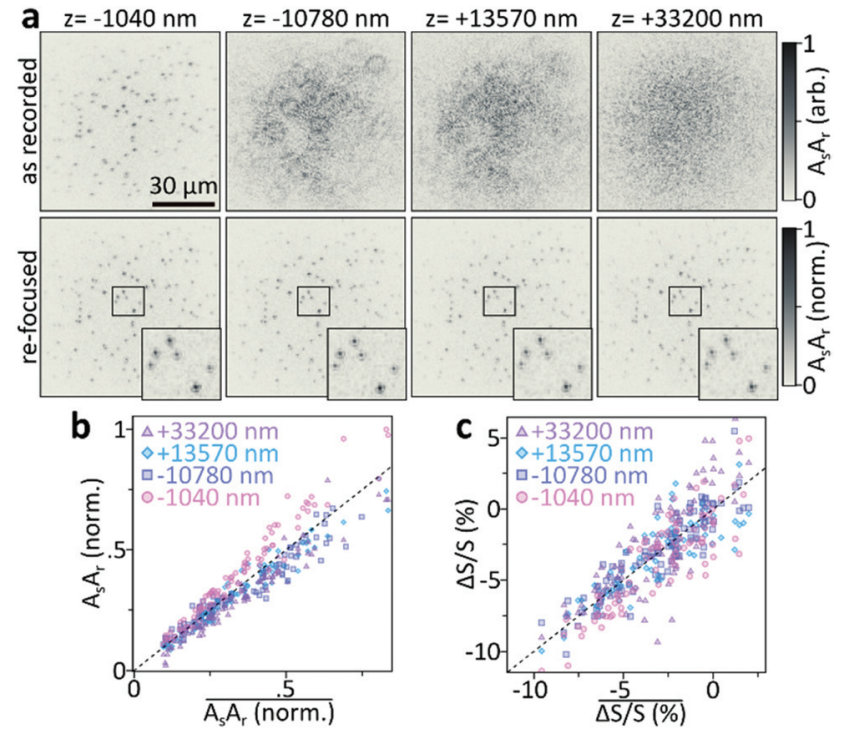

Fig. 4 Out-of-focus holographic and UHT imaging of $60 \mathrm{~nm}$ Au NPs. (a) Holographic images recorded at the same $x y$-sample-position but different $z$-defocus (top) compared to the images obtained after computational re-focusing (bottom). The re-focused images show nearly identical particle contrast and point-spread-function shape (inset). Only the image obtained after Fourier demodulation is shown. The refocused images are normalised to the same maximum value to allow quantitative comparison. (b) Correlation between the signal of each individual NP, obtained from the four recorded and re-focused images, and the mean-signal of the same NP. (c) As (b) but for the mean differential scattering $\Delta S / S$ in the $0-3.6$ ps pump-probe time-delay range. Pump: $400 \mathrm{~nm}$, probe: $550 \mathrm{~nm}$ wavelength.

exposure. Fig. 4a demonstrates these capabilities by imaging an out of focus 2D sample composed of many individual $60 \mathrm{~nm}$ diameter Au NPs, immobilised on a flat coverglass, at different defocus distances with respect to the image plane. For small defocus distances, similar to those employed in our proof-of-principle work $(z=-1040 \mathrm{~nm})$, we still observe many individual particles, which however blur into a speckle-like pattern as we move the sample further away from the image plane $(z=10.7-33.2 \mu \mathrm{m}$, Fig. 4a, top). Despite this apparent loss of information, computational re-focussing (Methods) of all four recordings results in nearly identical in-focus images (Fig. 4a, bottom).

To further quantify these observations, we compare the signals retrieved from the four $z$-positions. Fig. $4 \mathrm{~b}$ correlates the signal of each particle in all four images with the mean signal of the respective particle. Within our $z$-range, the scattering signal is essentially independent of the sample's position with respect to the focal plane of the imaging system. Even though this observation might seem surprising at first, the near-identical signal levels are expected if we consider the image-collection and re-focusing operation. Signal loss occurs if the detector fails to record the sample information and it is hence sufficient to employ a detector that is large enough to fully capture the defocused point-spread-functions of the individual particles. Even though defocusing lowers the detected 
photons per camera pixel, the interferometric detection methodology, with a reference wave that covers the entire detector, ensures camera operation at the shot-noise limit, thus preventing problems associated with entering the dark-noise regime of a camera. ${ }^{22}$ Computational re-focussing, a deconvolution operation, will hence always recover the in-focus NP-signal as long as their density is sufficiently low in the field of view and the signal-to-noise ratio is sufficiently high to observe the NP when physically in focus.

Thus far, we discussed imaging considerations as applied to 3D single-particle-localisation experiments with linear holography. However, the UHT microscope relies on signals recorded in a pump-probe fashion where an ultrafast pump pulse induces a time-dependent transient signal, or differential scattering $(\Delta S / S(t))$ change. Maximum UHT signals are generally obtained when probing the sample immediately after photoexcitation, ${ }^{10}$ maximising the magnitude of the photoinduced signal before the onset of relaxation processes. To demonstrate that UHT-based 3D single-particle-localisation experiments are indeed possible, we repeat the re-focusing experiments (Fig. 4a and b) but for differential scattering signals measured with the UHT microscope. A $400 \mathrm{~nm}$ pump pulse photoexcites the same set of $60 \mathrm{~nm}$ Au NPs and a $550 \mathrm{~nm}$ probe pulse captures the UHT-response as the average signal in the 0-3.6 ps time-window (computed from 31 individually acquired pump-probe delays). Fig. 4c, once again, shows that near-identical signals are obtained for in- and out-of-focus recordings, following digital refocusing. In other words, UHT microscopy should be well suited for 3D nonlinear localisation experiments over large volumes of view.

Following these static proof-of-concept experiments, we now concentrate on freely diffusing particles within a large 3D volume of $110 \times 110 \times 110 \mu \mathrm{m}$. Fig. 5 a shows a $3 \mathrm{D}$ representation of typically acquired linear holographic datasets where we combined results of three consecutively performed 3D tracking experiments on a total of 140 freely diffusing Au NPs. To obtain the trajectories shown we first acquire 60 holograms over a total observation time of $30 \mathrm{~s}$, using a camera exposure of $10 \mathrm{~ms}$ per image followed by a $500 \mathrm{~ms}$ waiting time. We then computationally propagate each hologram over a total of $150 \mu \mathrm{m}$, following the same procedure as previously described for the static samples (Fig. 4), and then localise all NPs in 3D (Methods), with localisation precisions typically being better than $15 \mathrm{~nm}$. The 3D coordinates of all particles in all 60 frames are then linked to obtain 3D trajectories using the procedure outlined in detail by Jaqaman et al. ${ }^{15,16,23}$

To demonstrate that the particles observed are, indeed, resonant $\mathrm{Au}$ NPs with their corresponding transient dynamics, and hence that $3 \mathrm{D}$ tracking can be applied to the transient signals, we follow a different approach. Rather than acquiring the entire video at a fixed pump-probe time-delay, with maximum NP-UHT response, we acquire consecutive images at either negative or positive pump-probe time-delays following the procedure described in Fig. 6a. In our approach, we first acquire a single UHT image at a positive pump-probe delay, using a $10 \mathrm{~ms}$ camera integration time. We then move to a negative delay and record a second image. The two acqui-

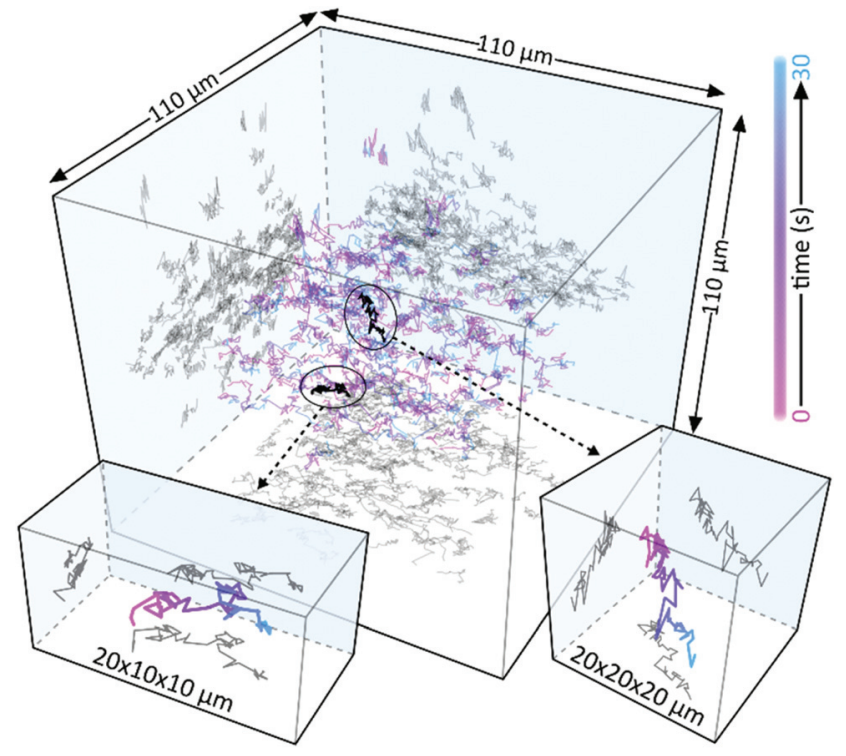

Fig. 5 Large volume-of-view holographic 3D tracking. (a) 3D representation of the trajectories of approximately $140 \mathrm{NPs}$ diffusing in aqueous solution. The pink-to-blue colour coding indicates time, coloured trajectories are 3D, grey trajectories the respective $x / y / z$ projections. Three individually recorded data-sets (each approximately $50 \mathrm{NPs}$ ) are combined for representation purposes. The two insets show representative, magnified, particle trajectories.

sitions are separated by a waiting time of $500 \mathrm{~ms}$ to allow sufficient time for physically moving the translation stage (Fig. 1a) that controls the pump-probe delay. In this experimental configuration we expect UHT signals at positive time delays and no signal at negative delays, which should allow us to demonstrate that the signal is photoinduced and not a potential artefact due to other experimental factors. Even though smaller particles are readily detectable ${ }^{10}$ we opted for rather large $100 \mathrm{~nm}$ Au NPs, with relatively slow diffusion coefficients, to ensure confident trajectory-linking even with the $500 \mathrm{~ms}$ waiting-time between frames. The differential scattering histograms reported in Fig. 6b show the absence of UHT signals for negative time delays and a marked signal increase at positive delays, thus verifying that UHT microscopy is capable of identifying resonant particles while, simultaneously, following their rapid 3D motion within a large volume of observation. As in the data reported in Fig. 4 we, once again, observe a large spread of UHT signals as the pump-beam's fluence is not constant across our large field of illumination. Fig. 6c shows examples of time-delay switching on the single-particle level where the individual NPs are centred and re-focused for representation purposes only. One particle is contained in the central area of the pump beam, corresponding to the peak of pump fluence, and the other in its spatial wing, in a region of negligible pump fluence. Even though both particles are visible in the pumpofF and pumpon images, only the NP in the high pump fluence area exhibits a UHT signal and only for positive time-delays. 
a
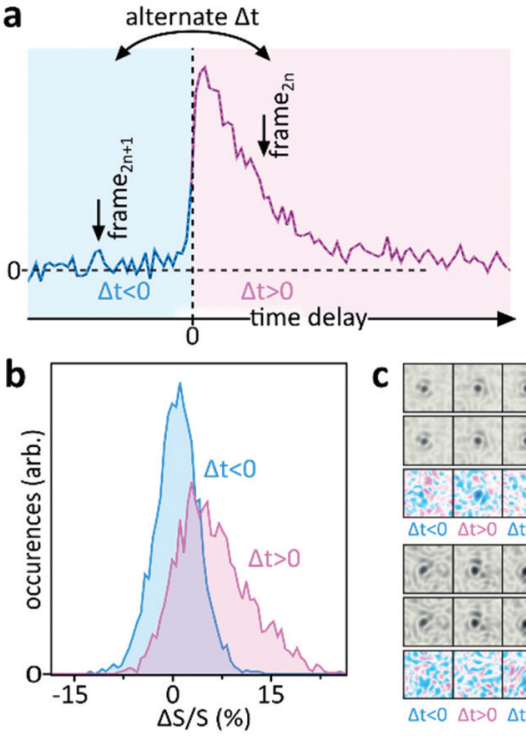
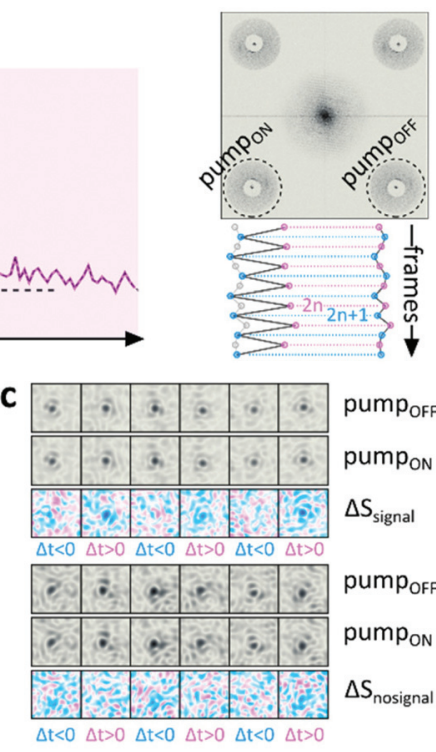

Fig. 6 In situ modulation of UHT signals for freely diffusing particles. (a) Alternating the pump-probe time-delay between image acquisitions switches the UHT signal on and off (left) which allows identifying resonant NPs as long as the same particle can be followed over several frames (right). (b) Comparison between the differential scattering signal histograms of all freely diffusing NPs acquired before (blue) and after (pink) photoexcitation. We note that the $\Delta S / S$ signal inversion, as compared to Fig. 4c, is due to the different size of the NPs, a different probe wavelength and the fact that the NPs are immersed in water $(n=1.334)$ rather than immobilized on a glass surface in air. (c) Comparison of pump $_{\mathrm{OFF}}$ and pump $\mathrm{ON}_{\mathrm{N}}$ images and difference between the two $(\Delta S)$ for a particle located in a high-fluence region (top) or a region of negligible pump-fluence (bottom). The freely diffusing particles are computationally re-focused and centred for representation purposes only, their physical position changes due to Brownian motion. Imaging is performed using a camera exposure of $10 \mathrm{~ms}$ followed by a $500 \mathrm{~ms}$ waiting-time to allow physical movement of the translation stage; pump: $400 \mathrm{~nm}$, probe: $566 \mathrm{~nm}$ wavelength.

We finally perform pump-probe time-dependent transient scattering spectroscopy on a freely diffusing $100 \mathrm{~nm}$ Au NP. Fig. 7a shows the as-recorded holographic image acquired with the focus placed in the vicinity of the glass-water inter- face. The two visible particles are stuck to the glass surface. We computationally propagate by approximately $10 \mu \mathrm{m}$ above the recording plane where we identify a freely diffusing NP (Fig. 7b, top). By continuously maximizing its amplitude via computational propagation, we are able to follow the $x y z-$ motion of said particle for holographic images acquired at different times, as shown in ESI Video 1. $\dagger$ Simultaneously, we record UHT images where the particle is only visible for positive time delays (Fig. $7 \mathrm{~b}$, bottom), in agreement with the observations made previously (Fig. 6). Finally, we use UHT microscopy to follow the 3D trajectory of the NP while changing the pump-probe time delay, which allows recording the $3 \mathrm{D}$ trajectory as well as the transient dynamics of a rapidly diffusing object (Fig. $7 \mathrm{c}$ and ESI video $1 \dagger$ ). As expected, the $\Delta S / S$ signal of the gold NP decays on the picosecond timescale due to hot electron cooling via coupling with phonons.

To summarise, we showed that UHT microscopy ${ }^{10}$ is ideally suited for single-particle tracking applications over large volumes of view. Analogous to photothermal microscopy, UHT microscopy allows identifying and isolating resonant particles, such as the gold NPs employed in this work, from off-resonant particles, which is the key requirement for imaging applications in scattering environments such as live cells or tissue. ${ }^{9}$ The holographic all-optical lock-in modality exploited in UHT microscopy further eliminates all limitations of traditional photothermal imaging, where lock-in amplifier based pointscanning severely limits the technique's applicability to observing dynamic systems, even in a simple $2 \mathrm{D}$ context. ${ }^{9}$ The relatively slow frame-rates of 2 frames-per-second employed here were solely chosen to allow changing the pump-probe time-delay between image acquisitions, which was necessary to conclusively demonstrate that the UHT signals observed are indeed pump-induced and not an artefact of our imaging system. For high-speed tracking applications the camera exposures of $10 \mathrm{~ms}$, used here, correspond to a nominal frame rate of 100 frames-per-second and it should easily be possible to further increase the frame rate to up to half the laser's repetition rate by employing a faster camera and further boost the sensitivity by using a laser with higher repetition rate. These capabilities make UHT microscopy an ideal candidate for both
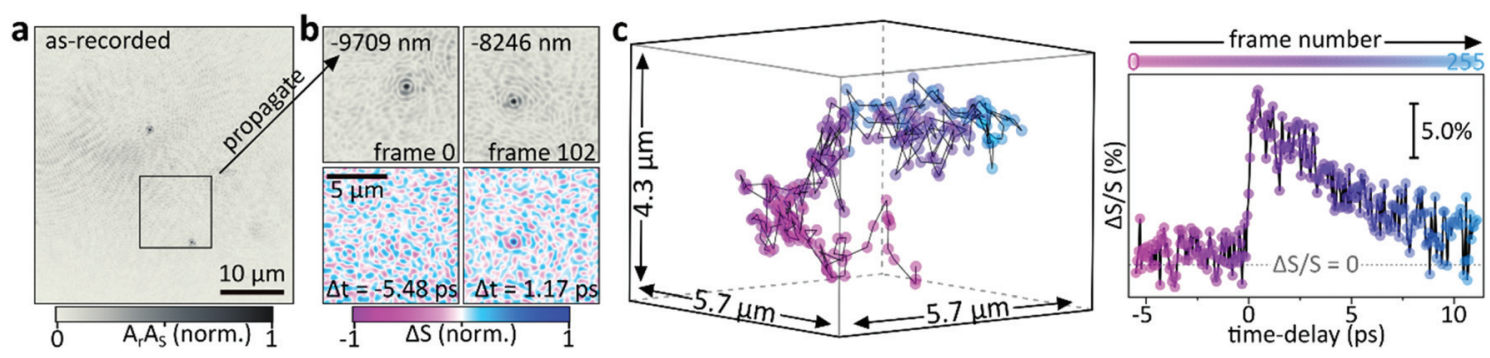

Fig. 7 Transient spectroscopy of freely moving objects. (a) As acquired image recorded at the glass-water interface highlighting the region of interest where a freely diffusing $100 \mathrm{~nm}$ Au NP is identified by (b) computationally propagating approximately $-9709 \mathrm{~nm}$ (frame 0 ) or $-8246 \mathrm{~nm}$ (frame 102). Transient UHT images obtained at the same frames but for two different pump-probe delays are shown below (see ESI Video $1 \dagger$ ). (c) $3 D$ trajectory of the freely diffusing NP shown in (a) alongside the simultaneously recorded transient scattering time-trace. 500 ms waiting-time between frames, $10 \mathrm{~ms}$ integration; pump: $400 \mathrm{~nm}$, probe: $566 \mathrm{~nm}$ wavelength. 
high-speed and long-term tracking of resonant particles in 3D scattering environments. As such, we envision immediate applications in the context of cellular particle-internalisation dynamics of theranostic or nanomedical agents as well as their transport through cells and tissue.

\section{Methods}

\section{Microscope}

The UHT microscope employed in this work is analogous to our previous implementation ${ }^{10}$ based on a $1 \mathrm{kHz}, 100 \mathrm{fs}$ amplified Ti:sapphire laser ${ }^{24}$ delivering both the $400 \mathrm{~nm}$ pump pulse as the second harmonic and the probe pulse via a homebuilt non-collinear optical parametric amplifier (NOPA). A 90:10 beam-splitter generates the probe and the reference from the NOPA output. A $F=200 \mathrm{~mm}$ achromatic lens focuses the collinear pump and probe pulses onto the sample. Residual pump light is removed with a $488 \mathrm{~nm}$ long-pass filter (488 $\mathrm{nm}$ EdgeBasic ${ }^{\mathrm{TM}}$, Semrock) and the probe scattering is collected with a home-built transmission dark-field microscope (NA $=0.5$, Olympus RMS20X-PF) and imaged onto a CMOS camera (acA2040-90um Basler ace, Basler AG) at a magnification of $37 \times$. Off-axis holography is implemented by interfering the sample scattering with two of the first diffraction orders of a $2 \mathrm{D} 0-\pi$ phase grating (25.6 grooves per $\mathrm{mm}$ ) which we relay-image (nominal magnification $0.5 \times$ ) onto the same CMOS camera. Two synchronized mechanical choppers are used to modulate both the reference waves as well as the pump pulse as described in detail previously ${ }^{10}$ and in the main text. The signal to reference time-delay is adjusted with a mechanical delay line. Pump-probe time-delay dependent images are recorded in an automatized fashion with a computer-controlled translation stage (M-531.PD1, Physik Instrumente).

\section{Sample preparation}

\#1.5 coverglass is cleaned by $10 \mathrm{~min}$ sonication in acetone, 10 min sonication in MilliQ, drying under a stream of $\mathrm{N}_{2}$ followed by 5 min oxygen plasma treatment (Femto, Diener electronic $\mathrm{GmbH}$ ). The cleaned glass is then incubated with PLL- $g$ PEG (SuSoS AG), for approximately $10 \mathrm{~min}$, followed by a dilute $60 \mathrm{~nm}$ Au NP-solution (BBI Solutions). The latex NPsample is fabricated by drying $5 \mu \mathrm{l}$ of $100 \mathrm{~nm}$ polystyrene particles (Sigma Aldrich) on glass. For experiments on freely diffusing particles we dilute the NP stock solution using MiliQ water containing $0.5 \%$ polyacrylic acid to reduce the NPs' mobility. The solutions are imaged using a coverglass sandwich with separation between the two glasses of $\approx 200 \mu \mathrm{m}$.

\section{Experimental parameters}

Due to the Gaussian beam-shapes the fluences vary widely across the field of observation and we solely quote $1 / e$ values for Fig. 4 and 5 with nominal fluences being: $1.00 \mathrm{~mJ} \mathrm{~cm} \mathrm{~cm}^{-2}$ pump, $0.75 \mathrm{~mJ} \mathrm{~cm}{ }^{-2}$ probe, $10 \mathrm{~ms}$ integration time.

\section{Angular spectrum method}

We perform image-propagation via the angular spectrum method. ${ }^{25}$ Briefly the processed $N x N$ holograms are convolved with a propagation kernel of the form:

$$
K(x, y, z)=\exp \left(i z \sqrt{k_{m}^{2}-k_{x}^{2}-k_{y}^{2}}\right),
$$

where $k_{m}=2 n \pi / \lambda$, with $n=1$ being the refractive index of air. The discretized spatial frequencies are $\left(k_{x}, k_{y}\right)=2 \pi / n \Delta x(x, y)$ for $(-N / 2 \leq x, y<N / 2)$, with $\Delta x$ representing the magnified pixel size of the imaging system.

\section{D localization, large volume}

For 3D localization, each hologram is propagated from $-75 \mu \mathrm{m}$ to $+75 \mu \mathrm{m}$ with a spacing between different $z$-planes $(\mathrm{d} z)$ of $500 \mathrm{~nm}$. The resulting image cube is then used to identify candidate particles by applying multiple convolutions in combination with noise and particle-size based thresholding. ${ }^{26}$ To achieve sub-pixel localization in the $x, y$-coordinates, particles that are in focus at a specific $z$-plane are fitted by a 2D Gaussian. For the $z$-coordinate, sub- $\mathrm{d} z$ localization is achieved by first calculating the Tamura values $(T(z)=\sqrt{\sigma(l z) / \text { mean }(l z)})$ for a region of interest of $(\approx 3 \times 3 \mu \mathrm{m})$, centred about the intensity maxima for each $z$-plane, and then fitting a parabola using the two most adjacent pixel values along the maximum. ${ }^{15}$

\section{Conflicts of interest}

There are no conflicts to declare.

\section{Acknowledgements}

The authors acknowledge support through the RTI2018099957-J-I00 and PGC2018-096875-B-I00 projects funded by MCIN/ AEI /10.13039/501100011033/ FEDER “A way to make Europe”, by the Ministry of Science and Innovations (MICINN "Severo Ochoa" program for Centers of Excellence in R\&D CEX2019-000910-S), the Catalan AGAUR (2017SGR1369), Fundació Privada Cellex, Fundació Privada Mir-Puig, and the Generalitat de Catalunya through the CERCA program. N.F.v. $\mathrm{H}$. acknowledges the financial support by the European Commission (ERC Advanced Grant 670949-LightNet). G.C. acknowledges support by the European Union Horizon 2020 Programme under Grant Agreement 881603 Graphene Core 3.

\section{References}

1 A. Gaiduk, M. Yorulmaz, P. V. Ruijgrok and M. Orrit, Room-Temperature Detection of a Single Molecule's Absorption by Photothermal Contrast, Science, 2010, 330(6002), 353-356.

2 K. Mawatari, T. Kitamori and T. Sawada, Individual Detection of Single-Nanometer-Sized Particles in Liquid by 
Photothermal Microscope, Anal. Chem., 1998, 70(23), 50375041, DOI: 10.1021/ac980250m.

3 D. Boyer, P. Tamarat, A. Maali, B. Lounis and M. Orrit, Photothermal Imaging of Nanometer-Sized Metal Particles among Scatterers, Science, 2002, 297(5584), 1160-1163, DOI: $10.1126 /$ science.1073765.

4 Y. C. Huang, T. H. Chen, J. Y. Juo, S. W. Chu and C. L. Hsieh, Quantitative Imaging of Single LightAbsorbing Nanoparticles by Widefield Interferometric Photothermal Microscopy, ACS Photonics, 2021, 8(2), 592602, DOI: 10.1021/acsphotonics.0c01648.

5 M. Tamamitsu, K. Toda, H. Shimada, T. Honda, M. Takarada, K. Okabe, Y. Nagashima, R. Horisaki and T. Ideguchi, Label-Free Biochemical Quantitative Phase Imaging with Mid-Infrared Photothermal Effect, Optica, 2020, 7(4), 359, DOI: 10.1364/optica.390186.

6 D. Zhang, C. Li, M. Slipchenko, C. Zhang and J. X. Cheng, Depth-Resolved Mid-Infrared Photothermal Imaging of Living Cells and Organisms at Sub-Micron Resolution, Sci. Adv., 2016, 2, e1600521, DOI: 10.1364/NTM.2017.NM4C.1.

7 S. Adhikari, P. Spaeth, A. Kar, M. D. Baaske, S. Khatua and M. Orrit, Photothermal Microscopy: Imaging the Optical Absorption of Single Nanoparticles and Single Molecules, ACS Nano, 2020, 14(12), 16414-16445, DOI: 10.1021/ acsnano.0c07638.

8 P. Vermeulen, L. Cognet and B. Lounis, Photothermal Microscopy: Optical Detection of Small Absorbers in Scattering Environments, J. Microsc., 2014, 254(3), 115-121, DOI: 10.1111/jmi.12130.

9 D. Lasne, G. A. Blab, S. Berciaud, M. Heine, L. Groc, D. Choquet, L. Cognet and B. Lounis, Single Nanoparticle Photothermal Tracking (SNaPT) of 5-Nm Gold Beads in Live Cells, Biophys. J., 2006, 91(12), 4598-4604, DOI: 10.1529/biophysj.106.089771.

10 M. Liebel, F. V. A. Camargo, G. Cerullo and N. F. van Hulst, Ultrafast Transient Holographic Microscopy, Nano Lett., 2021, 21, 1666-1671, DOI: 10.1021/acs.nanolett.0c04416.

11 P. Memmolo, L. Miccio, M. Paturzo, G. Di Caprio, G. Coppola, P. A. Netti and P. Ferraro, Recent Advances in Holographic 3D Particle Tracking, Adv. Opt. Photonics, 2015, 7(4), 713-755, DOI: 10.1364/AOP.7.000713.

12 L. Saemisch, N. F. Van Hulst and M. Liebel, One-Shot Phase Image Distinction of Plasmonic and Dielectric Nanoparticles, Nano Lett., 2021, 21, 4021-4028, DOI: 10.1021/acs.nanolett.1c00866.

13 M. Rubin, G. Dardikman, S. K. Mirsky, N. A. Turko and N. T. Shaked, Six-Pack off-Axis Holography, Opt. Lett., 2017, 42(22), 4611-4614.

14 P. Bon, G. Maucort, B. Wattellier and S. Monneret, Quadriwave Lateral Shearing Interferometry for Quantitative Phase Microscopy of Living Cells, Opt. Express, 2009, 17(15), 13080-13094, DOI: 10.1364/OE.17.013080.
15 M. Liebel, J. O. Arroyo, V. S. Beltrán, J. Osmond, A. Jo, H. Lee, R. Quidant and N. F. van Hulst, 3D Tracking of Extracellular Vesicles by Holographic Fluorescence Imaging, Sci. Adv., 2020, 6(45), eabc2508, DOI: 10.1126/SCIADV.ABC2508.

16 M. Liebel, N. Pazos-Perez, N. F. van Hulst and R. A. AlvarezPuebla, Surface-Enhanced Raman Scattering Holography, Nat. Nanotechnol., 2020, 15, 1005-1011, DOI: 10.1038/ s41565-020-0771-9.

17 A. A. Maznev, T. F. Crimmins and K. A. Nelson, How to Make Femtosecond Pulses Overlap, Opt. Lett., 1998, 23(17), 1378-1380, DOI: 10.1364/OL.23.001378.

18 M. Takeda, H. Ina and S. Kobayashi, Fourier-Transform Method of Fringe-Pattern Analysis for Computer-Based Topography and Interferometry, J. Opt. Soc. Am., 1982, 72(1), 156, DOI: 10.1364/JOSA.72.000156.

19 E. Cuche, P. Marquet and C. Depeursinge, Spatial Filtering for Zero-Order and Twin-Image Elimination in Digital offAxis Holography, Appl. Opt., 2000, 39(23), 4070-4075, DOI: 10.1364/AO.39.004070.

20 C. Pache, N. L. Bocchio, A. Bouwens, M. Villiger, C. Berclaz, J. Goulley, M. I. Gibson, C. Santschi and T. Lasser, Fast Three-Dimensional Imaging of Gold Nanoparticles in Living Cells with Photothermal Optical Lock-in Optical Coherence Microscopy, Opt. Express, 2012, 20(19), 21385, DOI: $10.1364 /$ oe.20.021385.

21 A. Block, M. Liebel, R. Yu, M. Spector, Y. Sivan, F. J. García De Abajo and N. F. Van Hulst, Tracking Ultrafast HotElectron Diffusion in Space and Time by Ultrafast Thermomodulation Microscopy, Sci. Adv., 2019, 5(5), eaav8965, DOI: 10.1126/sciadv.aav8965.

22 U. Ortiz-Orruño, A. Jo, H. Lee, N. F. Van Hulst and M. Liebel, Precise Nanosizing with High Dynamic Range Holography, Nano Lett., 2021, 21(1), 317-322, DOI: 10.1021/ acs.nanolett.0c03699.

23 K. Jaqaman, D. Loerke, M. Mettlen, H. Kuwata, S. Grinstein, S. L. Schmid and G. Danuser, Robust Single-Particle Tracking in Live-Cell Time-Lapse Sequences, Nat. Methods, 2008, 5(8), 695-702, DOI: 10.1038/nmeth.1237.

24 C. Manzoni, D. Polli and G. Cerullo, Two-Color Pump-Probe System Broadly Tunable over the Visible and the near Infrared with Sub-30 Fs Temporal Resolution, Rev. Sci. Instrum., 2006, 77(2), 023103, DOI: 10.1063/ 1.2167128 .

$25 \mathrm{~J}$. W. Goodman, Introduction to Fourier Optics, ed. W. H. Freeman, 3rd edn, 2005.

26 P. Viola and M. Jones, Rapid Object Detection Using a Boosted Cascade of Simple Features. In Proceedings of the 2001 IEEE Computer Society Conference on Computer Vision and Pattern Recognition. CVPR 2001, Kauai, HI, USA, 2001; 2005; pp I-511-I-518. DOI: 10.1109/ cvpr.2001.990517. 